\title{
Erratum to: Identification of Conjugated Pentadecadienals as a Sex Pheromone Components of the Sphingid Moth, Dolbina tancrei
}

\author{
Takuya Uehara • Hideshi Naka • Shigeru Matsuyama • \\ Le van Vang • Tetsu Ando • Hiroshi Honda
}

Published online: 2 September 2014

(C) Springer Science+Business Media New York 2014

Erratum to: J Chem Ecol (2013) 39: 1441-1447.

DOI 10.1007/s10886-013-0357-1

The original version of this article contained a mistake. In the extraction of sex pheromone, the correct calling time was in the first 3 to $6 \mathrm{~h}$ of the "dark period", and not in the "light period". The authors regret this error.

The online version of the original article can be found at http://dx.doi.org/ 10.1007/s10886-013-0357-1.

\section{T. Uehara}

Graduate School of Life and Environmental Sciences,

University of Tsukuba, Tsukuba, Ibaraki 305-8572, Japan

H. Naka

Faculty of Agriculture, Tottori University, Tottori Koyama

Minami 680-8553, Japan

S. Matsuyama $\cdot$ H. Honda $(\bowtie)$

Faculty of Life and Environmental Sciences, University of Tsukuba,

Tsukuba, Ibaraki 305-8572, Japan

e-mail: honda.hiroshi.ew@u.tsukuba.ac.jp

L. van Vang $\cdot$ T. Ando

Graduate School of Bio-Applications and Systems Engineering,

Tokyo University of Agriculture and Technology,

Koganei 184-8588, Japan 